\title{
PRONE TO SUPINE SURFACE BASED REGISTRATION WORKFLOW FOR BREAST TUMOR LOCALIZATION IN SURGICAL PLANNING
}

\author{
F. Alfano ,JE. Ortuño Fisac , Mónica Garcia-Sevilla , M. Herrero Conde , O. Bueno Zamora , \\ S. Lizarraga , A. Santos , J. Pascau , MJ. Ledesma Carbayo
}

\begin{abstract}
Breast cancer is the most frequent cancer in women worldwide. Screening programs and imaging improvements have increased the detection of clinically occult nonpalpable lesions requiring preoperative localization. Imageguided wire localization (WGL) is the current standard of care for the excision of non-palpable carcinomas during breast conserving surgery (BCS). Due to the current limitations of intraoperative tumor localization approaches, the integration of the information from multimodal imaging may be especially relevant in surgical planning. This work presents a workflow to perform a prone image-to-surgical physical data alignment in order to determine the correspondence between the tumor identified in the preoperative image and the final position of the tumor in the surgical position. The evaluation of the methodology has been carried out in 18 cases achieving an average localization error of $10.40 \mathrm{~mm}$ and $9.84 \mathrm{~mm}$ in 11 small lesion cases (less than $1 \mathrm{~cm}$ in diameter).
\end{abstract}

Index Terms - Surface registration, breast imaging, surgical planning

\section{INTRODUCTION}

Worldwide, there will be about 2.1 million newly diagnosed female breast cancer cases in 2018, representing almost 1 case of cancer among 4 women. The disease is the most frequently diagnosed cancer in the vast majority of countries and is also a leading cause of cancer death [1].

Screening programs and imaging improvements have increased the detection of clinically occult non-palpable breast lesions requiring preoperative localization [2,3]. In women with non-palpable breast cancer, several randomized trials have shown that breast conservative surgery is the treatment of choice [4]. The main challenge in the resection of non-palpable tumors is to obtain free margins by minimizing the resection of healthy breast tissue with good aesthetic results. Currently, wire-guided localization
(WGL) is the most widely used method for locating nonpalpable breast lesions. The limits of WGL include technical complications such as wire transection and migration, patient discomfort and poor cosmetic outcome $[5,6]$. The female breast undergoes different deformations due to external forces or changes in position during routine medical imaging. A prone MRI acquisition provides an accurate delineation of the size and extent of the tumor and offers the highest sensitivity for intraductal extensions typical of invasive cancers. However, while prone breast MRI is well suited for diagnosis, it is less suitable for guiding a breast conservative surgery where the patient typically lies in a supine position, with the arm extended. Hence, breast lesions undergo significant displacements between the preoperative image and intraoperative positions. Due to the current limitations of intraoperative tumor localization approaches, several groups have investigated the use of preoperative MR images acquired in positions closer to the surgical orientation. Methods using biomechanical models for prone-to-supine registration of MR images for use in image-guided breast surgery have been suggested [7,8]. Registration of preoperative supine MR images to physical space in the operating room OR for MRI navigated breast surgery has been also described [9]. Preoperative supine MR images rigidly registered with an intraoperative optical scan to adjust the MRI image to the exact breast position in the OR have also been used for surgical guidance [10]. A proneto-supine surface registration and a localization method based on the transfer of surface displacements to the tumor has been considered in our previous work, achieving good results but being sensitive to the tumor size [11].

This work presents a workflow to perform a prone image-tosurgical physical data alignment strategy for determining the correspondence between the tumor identified in the preoperative image and the final position of the tumor in the surgical position. The developed method is based only on the prone MRI volume and the surface of the patient in the surgical orientation. The tumor localization error has been evaluated using retrospective cases with preoperative prone MRI and supine surface extracted from a CT image. The 
position of the tumor has been estimated using a workflow that combines a Laplacian deformation, followed by an iterative point registration to refine the prone-to-supine surface matching and a final surface driven B-spline registration to derive the dense displacement field in the whole breast. This approach has been tested in 18 clinical cases with tumor lesions of different sizes and compared with our previous method [11].

\section{MATERIALS AND METHODS}

The aim of this work is to provide an alternative or complementary tool to pre-surgical localization techniques of non-palpable breast lesions without altering the diagnostic imaging protocol by using a prone preoperative MRI and the surface in the surgical position. The implemented system estimates the deformation occurred in the volume of the breast starting from the pose change transformation of the surface of the breast and identifies the final position of the tumor. To perform a demonstration and evaluation of the concept, preoperative MR and CT images were collected to extract the breast surface, respectively, in the prone and supine positions and the tumor was segmented in both images. The pose transformation from prone to supine has been performed to calculate the correspondence point-to-point between the initial mesh and the final mesh in order to derive a dense displacement field using a point driven B-spline registration. The whole workflow is described in Figure 1. To validate the results the distance between the supine tumor centroid identified in the CT image and the tumor centroid estimated with the implemented technique has been considered.
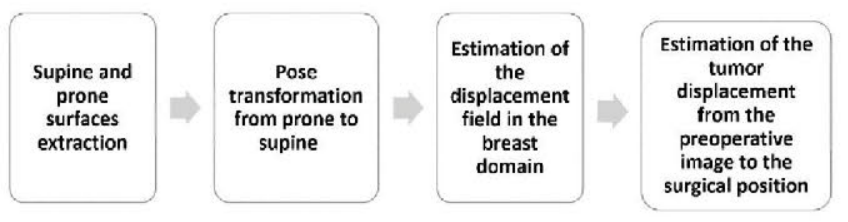

Figure 1. Workflow: upon the prone and surface generation, the pose transformation of pose is performed and the displacement field in the breast domain is estimated to predict the position of the tumor in the intraoperative position.

\subsection{Data}

The data includes 18 retrospective cases of breast lesions, 11 of which are under $1 \mathrm{~cm}$ in diameter, provided by the General University Hospital Gregorio Marañón (HGUGM) in Madrid. Each case consists of a prone MRI study including an MRI T2 SPAIR and a Subtraction MRI and a supine CT study.

Since the diagnostic hospital protocol does not involve a supine MRI image, staging CT scans in supine position have been used to simulate the intraoperative surface. Table 1 reports the range of lesion's diameters, lesion to skin distances, and total prone-to-supine lesion displacement for the 18 cases considered in this work. It is important to underline that the acquisition of a CT image does not belong to the standard preoperative protocol but is required when it is needed for cancer staging purposes. This fact limits the number of cases available. Only the surface of the breast derived from the $\mathrm{CT}$ image is used in the proposed approach.

\begin{tabular}{|c|c|c|}
\hline $\begin{array}{c}\text { Diameters } \\
\text { [mm] }\end{array}$ & $\begin{array}{c}\text { Distance to skin } \\
\text { [mm] }\end{array}$ & $\begin{array}{c}\text { Total displacement from } \\
\text { prone to supine position [mm] }\end{array}$ \\
\hline $5.51-23.10$ & $11.23-35.25$ & $32.78-118.61$ \\
\hline
\end{tabular}

Table 1. Range of lesion's diameter, lesion to skin distances, and prone-to-supine lesion displacement. The tumor diameter has been measured as the maximum diameter of the lesion in axial planes of the supine MRI image.

\subsection{Data processing}

Upon the segmentation of the breast tissue in the MR and CT images, a 3D triangular mesh representing the entire surface of the breast volume in the prone configuration and a 3D surface mesh representing the skin of the breast in the supine surgical orientation have been generated using iso2mesh [12]. Due to the low quality of the MRI T2 SPAIR, an automatic segmentation of the chest wall was not possible instead, an approximate segmentation has been considered as shown in Figure 2. The surface of the posterior limit of the breast was established as a condition of zero displacement during the transformation of pose from prone to supine [9].

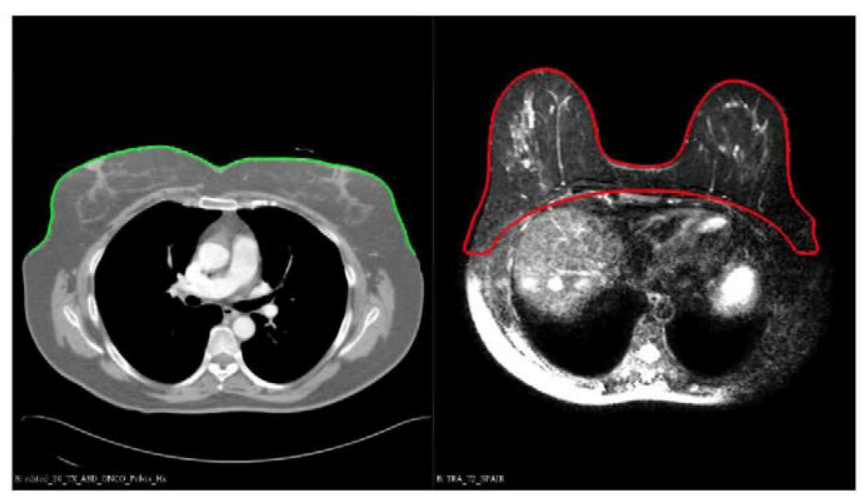

Figure 2. CT image in supine position and the extracted skin surface as a triangular mesh (green). MRI T2 SPAIR image in prone position and the extracted surface of the breast volume as a triangular mesh (red).

\subsection{Pose transformation}

The female breast undergoes large deformations when its pose changes from prone to supine as it possible to appreciate in Figure 2. Hence, the pose transformation has been performed in two steps. The first step goal is to perform a coarse transformation using a Laplacian deformation given a set of vertices with prescribed displacement [13]. This deformation considers that the 
anatomical axes starting from the nipples do not vary between prone and supine positions. The vertices with prescribed displacements have been automatically calculated on the two surfaces starting from 3 anatomical points (nipples and suprasternal notch). The Laplacian deformation method is described in more detail in [11]. As a second step, a non-rigid ICP algorithm is applied to refine the mapping of the prone skin surface onto the target surface. The aim of these 2-steps is to obtain a dense pointto-point matching of the two surfaces to enable the computation of the whole breast deformation based on a point-matching B-spline deformation. Figure 3.b and 3.c show the final deformed surface versus the prone source surface and the target surface.

a)

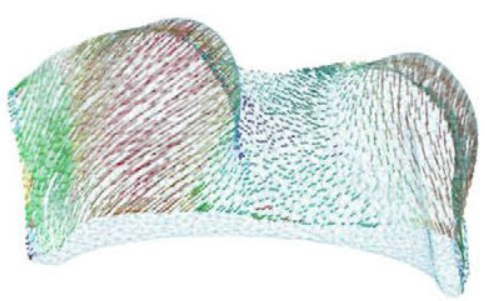

b)

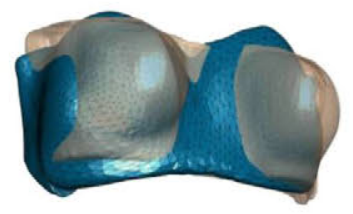

c)

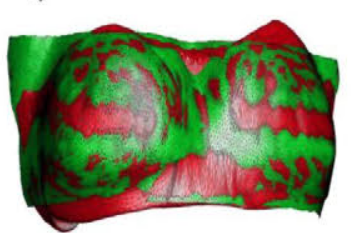

Figure 3. (a) Arrow vectors illustrating the displacement magnitude distribution on the prone surface. (b) Superposition of the prone (transparent orange) and the final deformed surface. (c) Superposition of the supine target surface (green) and the final deformed surface (red)

\subsection{Tumor Localization}

The main idea underlying the final tumor position estimation technique is to smoothly propagate the transformation associated with the surface data onto all the positions in the breast domain in order to be evaluated at any desired set of positions.

The surface transformation captures the global motion of the breast, to predict the final position of the tumor inside the volume a smooth and consistent deformation field of the breast tissue is needed.

Free form deformation FFD model based on B-splines have been successfully employed in nonrigid registration of medical images [14]. The basic idea of FFDs is to deform an object by manipulating an underlying mesh of uniformly spaced control points. The deformation of the mesh controls the shape of the object or image we want to deform to match another object or image, in our case the two corresponding surface points.

Our approach to achieve the displacements occurring into the breast domain is based on a B-spline based free-form deformation (FFD) algorithm proposed in $[14,15]$ which the total transformation model is represented by $\boldsymbol{T}_{\text {local }}$, a $3 \mathrm{D}$ tensor product of the familiar 1D cubic B-splines:

$T_{\text {local }}(x, y, z)=\sum_{l=0}^{3} \sum_{m=0}^{3} \sum_{n=0}^{3} B_{l}(u) B_{m}(v) B_{n}(w) \Phi_{\mathrm{i}+\mathrm{l}, \mathrm{j}+\mathrm{m}, \mathrm{k}+\mathrm{n}}$

where $\Phi$ denotes the unknown values for a number of control points that form a regular lattice of size $n_{x} \times n_{y} \times n_{z}, i$, $j, k$ are the indexes of the control points, and $u, v, w$ correspond to the relative position of $(x, y, z)$ in the lattice coordinates and where $B_{i}$ represents the $l_{t h}$ basis function of the B-spline.

The optimization metric is based on the quadratic error of the points of the supine surface with respect to the progressively deformed prone surface. Apart from the breast surfaces several corresponding points of the chest wall are also considered as fixed, to ensure that the estimation of the motion near the back surface is not affected. A multiresolution approach in the deformation model is followed to approximate the deformation in a coarse to fine fashion ranging from a grid of control points of $8 \times 8 \times 8$ to $68 \times 68 \times 68$.

The final tumor position has been computed by applying the obtained non-rigid transformation to the tumor points in the prone position. This localization methodology has been compared with the previously described transfer of surface displacement method [11].

\section{RESULTS}

Figure 4 shows the distance between the centroid of the predicted tumor location and the centroid of the segmented tumor in the $\mathrm{CT}$ image.

The transfer of surface displacements method (TSD) proposed in [11] with the new set of data shows a mean error of 10.24 which is slightly lower than the one obtained with the proposed method based on B-splines $(10.40 \mathrm{~mm})$. However, the B-spline based method (BSM) shows better results localizing small lesions (less than $1 \mathrm{~cm}$ in diameter).

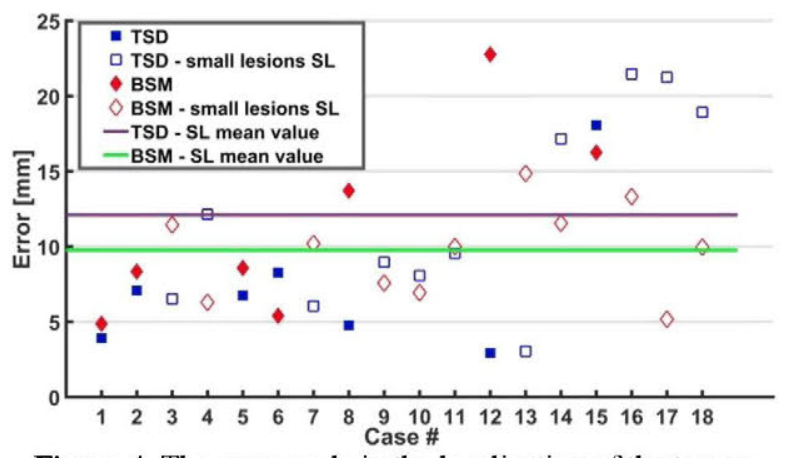

Figure 4. The error made in the localization of the tumor.

As shown in Figure 4, in these cases the BSM achieves a mean error of $9.84 \mathrm{~mm}$ versus the $12.10 \mathrm{~mm}$ of the TSD method. Figure 5 shows the visual results for case 4 . 


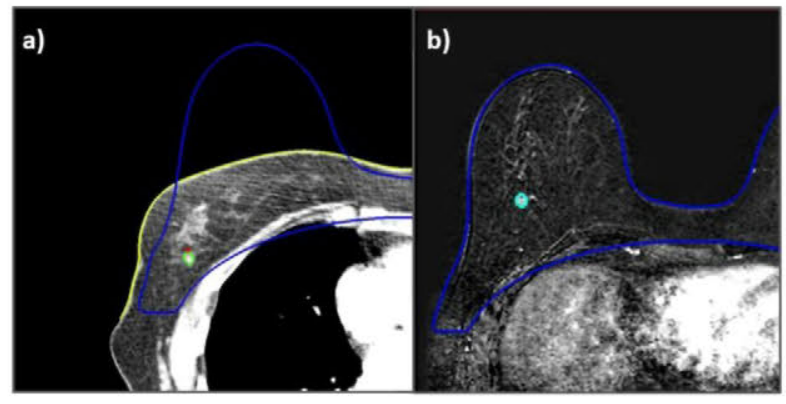

Figure 5. Results of case 4: a) supine image and surface (yellow), prone surface (blue), estimated tumor (red) and real tumor in supine position (green). b) prone image and prone surface (blue), real tumor in prone position (light blue).

\section{CONCLUSIONS}

This work proposes and validates a method to estimate small breast lesions localization in the surgery physical space, starting from a preoperative MR image acquired in prone position and a surface acquisition in surgical position. The results, calculated as distances between the predicted location of the tumor and the tumor segmented in the supine image in 18 cases, show a mean error of $10.40 \mathrm{~mm}$ and of $9.84 \mathrm{~mm}$ in the cases of lesions under $1 \mathrm{~cm}$ in diameter. Guiding is especially necessary for small non-palpable lesions. Similar results have been achieved with other proposals of the literature that require a supine MRI acquisition using image-based registration techniques [10] or that rely on finite element analysis [8]. In comparison with the previously proposed transfer of surface displacement method, the proposed approach is independent of the size of the tumor and avoids the retroprojection step to locate the tumor that is computationally expensive. The proposed methodology is additionally very efficient

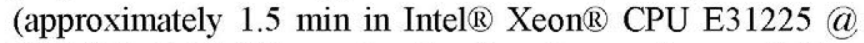
$3.10 \mathrm{GHz} 3.10 \mathrm{GHz}$ and as it only relies on the acquisition of the intraoperative surface of the patient, it is electable to be used for computing the localization of the tumor in the OR to aid in the surgical procedure.

The validation of the current proposal has been developed using the available information closest to the surgical position (a supine CT). Using an optical scanner would allow the acquisition of the breast surface in the operating room, altering minimally the surgical procedure. This acquisition would replace the surface obtained from the CT scan used for validation purposes in this work.

Future work will consider the introduction of material properties constraints obtained automatically from the MRI volume of the breast to improve the results and the incorporation of deep learning techniques to learn the deformation field [16] underlying the transformation from preoperative to intraoperative pose.

\section{ACKNOWLEDGMENT}

This study was supported by projects TEC2015-66978-R, DTS14/00192, PI-15/02121 (Ministerio de Economía y Competitividad, ISCIII), TOPUS-CM S2013/MIT-3024 (Comunidad de Madrid) and FEDER funds.

\section{REFERENCES}

[1] F. Bray, et al. "Global cancer statistics 2018: GLOBOCAN estimates of incidence and mortality worldwide for 36 cancers in 185 countries," CA: a cancer journal for clinicians, 2018.

[2] KA. Skinner, et al. "Palpable breast cancers are inherently different from nonpalpable breast cancers," Annals of Surgical Oncology, 8(9), pp. 705-710, 2001.

[3] B. Cady, et al. "The new era in breast cancer. Invasion, size, and nodal involvement dramatically decreasing as a result of mammographic screening," Atomic Spectroscopy, 131(3), pp. 301308, 1996.

[4] U. Veronesi, et al. "Twenty-year follow-up of a randomized study comparing breast-conserving surgery with radical mastectomy for early breast cancer," New England Journal of Medicine, 347(16), pp. 1227-1232, 2002.

[5] P. S. Davis, et al. "Migration of breast biopsy localization wire," American Journal of Roentgenology, 150.4, pp. 787-788, 1988.

[6] A. Muneer, et al. "Systematic review of radioguided versus wire-guided localization in the treatment of non-palpable breast cancers," Breast cancer research and treatment, 140.2, pp. 241252, 2013.

[7] T. J. Carter, et al. "MR navigated breast surgery: method and initial clinical experience," Medical Image Computing and Computer-Assisted Intervention-MICCAI 2008, pp. 356-363. Springer, 2008.

[8] L. Han, et al., "A nonlinear biomechanical model-based registration method for aligning prone and supine MR breast images," IEEE transactions on medical imaging, 33(3), pp. 682694, 2014.

[9] T. J. Carter, et al., "A framework for image-guided breast surgery," International Workshop on Medical Imaging and Virtual Reality, pp. 203-210, 2006.

[10] M. J. Pallone, et al., "Supine breast MRI and 3D optical scanning: A novel approach to improve tumor localization for breast conserving surgery," Annals of surgical oncology, 21.7, pp. 2203-2208, 2014.

[11] Alfano, F., et al. "Tumor localization using prone to supine surface-based registration for breast cancer surgical planning," Biomedical Imaging (ISBI 2018), 2018 IEEE 15th International Symposium on (pp. 788-791), IEEE, 2018.

[12] Fang, Q., et al. "iso2mesh: a 3D surface and volumetric mesh generator for MATLAB." Octave, Erişim: http://iso2mesh. sourceforge. net, 2010.

[13] M. Botsch and O. Sorkine, "On linear variational surface deformation methods," IEEE transactions on visualization and computer graphics, vol. 14, pp. 213-230, 2008.

[14] D. Rueckert, et al., "Nonrigid registration using free-form deformations: application to breast MR images," IEEE transactions on medical imaging, 18(8), pp. 712-721, 1999.

[15] J. Kybic, and M. Unser. "Fast parametric elastic image registration.", 2003.

[16] G. Balakrishnan, et al. "VoxelMorph: A Learning Framework for Deformable Medical Image Registration," arXiv preprint arXiv:1809.05231, 2018. 